\title{
Moyamoya Syndrome after Radiation Therapy: a Clinical Report
}

\author{
Paulo Almeida ${ }^{1}$, Ana Luísa Rocha ${ }^{2,3}$, Gonçalo Alves ${ }^{4}$, Tiago Parreira ${ }^{4}$, Maria Luís Silva ${ }^{4}$, \\ António Cerejo ${ }^{3,5}$, Pedro Abreu ${ }^{2,3}$, Ana Monteiro ${ }^{3,6}$ \\ ${ }^{1}$ Department of Internal Medicine, Centro Hospitalar Baixo Vouga, Aveiro, Portugal \\ ${ }^{2}$ Department of Neurology, Centro Hospitalar Universitário São João, Porto, Portugal \\ ${ }^{3}$ Clinical Neurosciences and Mental Health Department, Faculty of Medicine of the University of Porto, Portugal \\ ${ }^{4}$ Department of Neuroradiology, Centro Hospitalar Universitário São João, Porto, Portugal \\ ${ }^{5}$ Department of Neurosurgery, Centro Hospitalar Universitário São João, Porto, Portugal \\ ${ }^{6}$ Department of Neurology, Unidade Local de Saúde de Matosinhos, Matosinhos, Portugal
}

\section{Doi: 10.12890/2019_001337 - European Journal of Case Reports in Internal Medicine - ๑ EFIM 2019}

Received: 17/10/2019

Accepted: 23/10/2019

Published: 02/12/2019

How to cite this article: Almeida P, Rocha AL, Alves G, Parreira T, Silva ML, Cerejo A, Abreu P, Monteiro A. Moyamoya syndrome after radiation therapy: a clinical report. EJCRIM 2019;6: doi:10.12890/2019_001337.

Conflicts of Interests: The Authors declare that there are no competing interest

This article is licensed under a Commons Attribution Non-Commercial 4.0 License

\section{ABSTRACT}

Moyamoya syndrome (MMS) is a rare, chronic and progressive vasculopathy with a characteristic angiographic pattern and well-recognized predisposing conditions, such as cranial therapeutic radiation. We report the case of a 36-year-old Caucasian female with a history of craniopharyngioma treated with whole-brain radiotherapy 20 years previously. She was admitted to the emergency department with disorientation and imperceptible speech lasting for 1 hour. Upon examination, she had slight motor aphasia, without sensory or motor deficits. However, the neurological deficits worsened on standing position. The computed tomography (CT) angiogram and transcranial Doppler ultrasonography revealed occlusion of the distal portion of the left internal carotid artery (ICA). Mechanical thrombectomy was attempted without success. Head CT was repeated, revealing left periventricular acute ischaemic stroke. The cerebral angiography showed total occlusion of the left ICA with an exuberant network of transdural collateral vessels, confirming MMS. The patient completed a functional rehabilitation program with progressive improvement of deficits and maintained a multidisciplinary follow-up. MMS is a serious late complication from cranial radiation therapy and a well-described risk factor for ischaemic stroke in younger patients. Therefore, early detection and prompt treatment are mandatory, as the consequences can be disastrous, such as cognitive and neurologic decline due to repeated strokes.

\section{LEARNING POINTS}

- Moyamoya syndrome should be considered in younger patients presenting with acute neurologic deficits, particularly those treated with cranial radiotherapy.

- The treatment of acute stroke in patients with moyamoya syndrome should include intravenous hydration and avoidance of hypotension.

- The benefit of antiplatelet agents is limited and equivocal for patients with moyamoya syndrome and oral anticoagulants are not recommended for long-term treatment.

\section{KEYWORDS}

Radiotherapy, moyamoya syndrome, craniopharyngioma 


\section{CASE DESCRIPTION}

We report the case of a Caucasian female with a history of craniopharyngioma at the age of 16 , treated with surgery and adjuvant whole-brain radiotherapy. Despite a good outcome without signs of relapse, there were some sequelae, including panhypopituitarism, severe bilateral hypovision, right hearing loss and developmental delay with limited functional status (modified Rankin scale score of 3/6). She presented at our emergency department at the age of 36 with disorientation and imperceptible speech lasting for 1 hour. Upon examination, she had slight motor aphasia, scoring 2 on the National Institutes of Health Stroke Scale (NIHSS). Laboratory studies revealed no changes. Non-contrast computed tomography (CT) of the head revealed no acute ischaemic lesions or intracerebral haemorrhage. Neurological deficits worsened after approximately 2 to 3 minutes of standing up, with left-sided hemiparesis, mild left sensory loss and dysarthria (NIHSS score of 6). The neurological deficits improved after lying down. A CT angiogram revealed occlusion of the distal portion of the left internal carotid artery (ICA) up to the proximal segments of the ipsilateral middle (MCA) and anterior (ACA) cerebral arteries, as well as diffuse reduction in the calibre of the right ICA, more markedly in the intracranial portion. Transcranial Doppler ultrasonography (TCD) confirmed occlusion of the distal portion of the left ICA and showed signs of collateralization to the right carotid territory via the ipsilateral posterior communicating artery (Fig. 1). Mechanical thrombectomy was attempted without success since the guidewire could not go through the left ICA after the emergence of the ophthalmic artery.

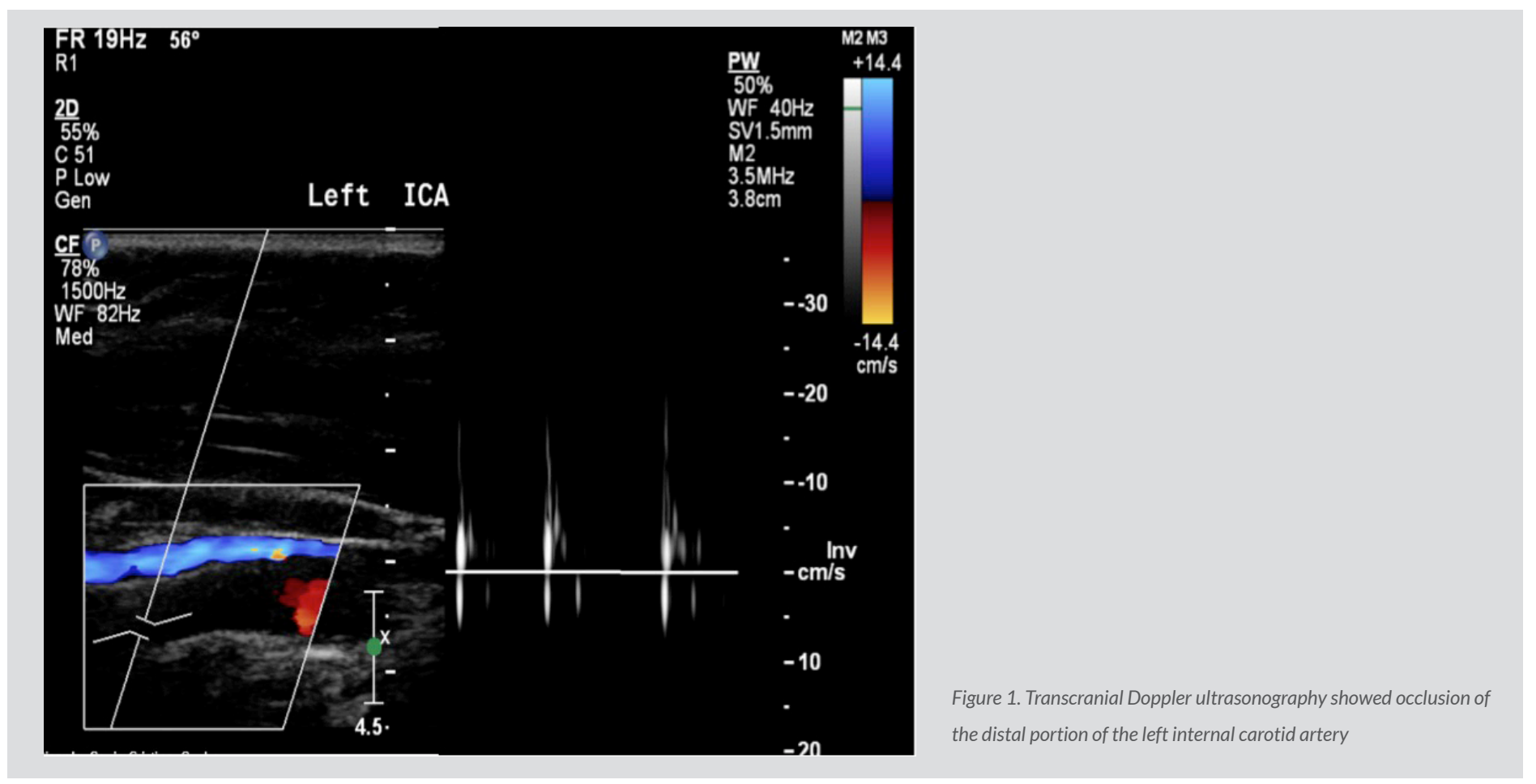

Mechanical thrombectomy was attempted without success since the guidewire could not go through the left ICA after the emergence of the ophthalmic artery.

The patient was admitted to the Stroke Unit. Control head CT revealed acute left periventricular ischaemic stroke. An echocardiogram, continuous heart rate monitoring and extensive laboratory studies were unrevealing. Cerebral angiography was performed and confirmed the findings of total occlusion of the left ICA with multiple leptomeningeal collateral vessels of the left posterior cerebral artery filling retrogradely the territory of the left MCA (Fig. 2). Furthermore, cerebral angiography revealed right ICA occlusion after the emergence of the anterior choroidal artery and an abnormal network of transdural collateral vessels that filled retrogradely the territory of the right MCA and ACA (Fig. 3). These angiographic findings are characteristic of bilateral moyamoya syndrome (MMS). The patient was started on platelet antiaggregation treatment and a statin. During her hospital stay, she completed a functional rehabilitation program with progressive improvement of deficits. She was discharged with an NIHSS score of 3. The patient maintained a multidisciplinary follow-up (Physiatry, Neurology and Neurosurgery), and revascularization surgery is being considered. 

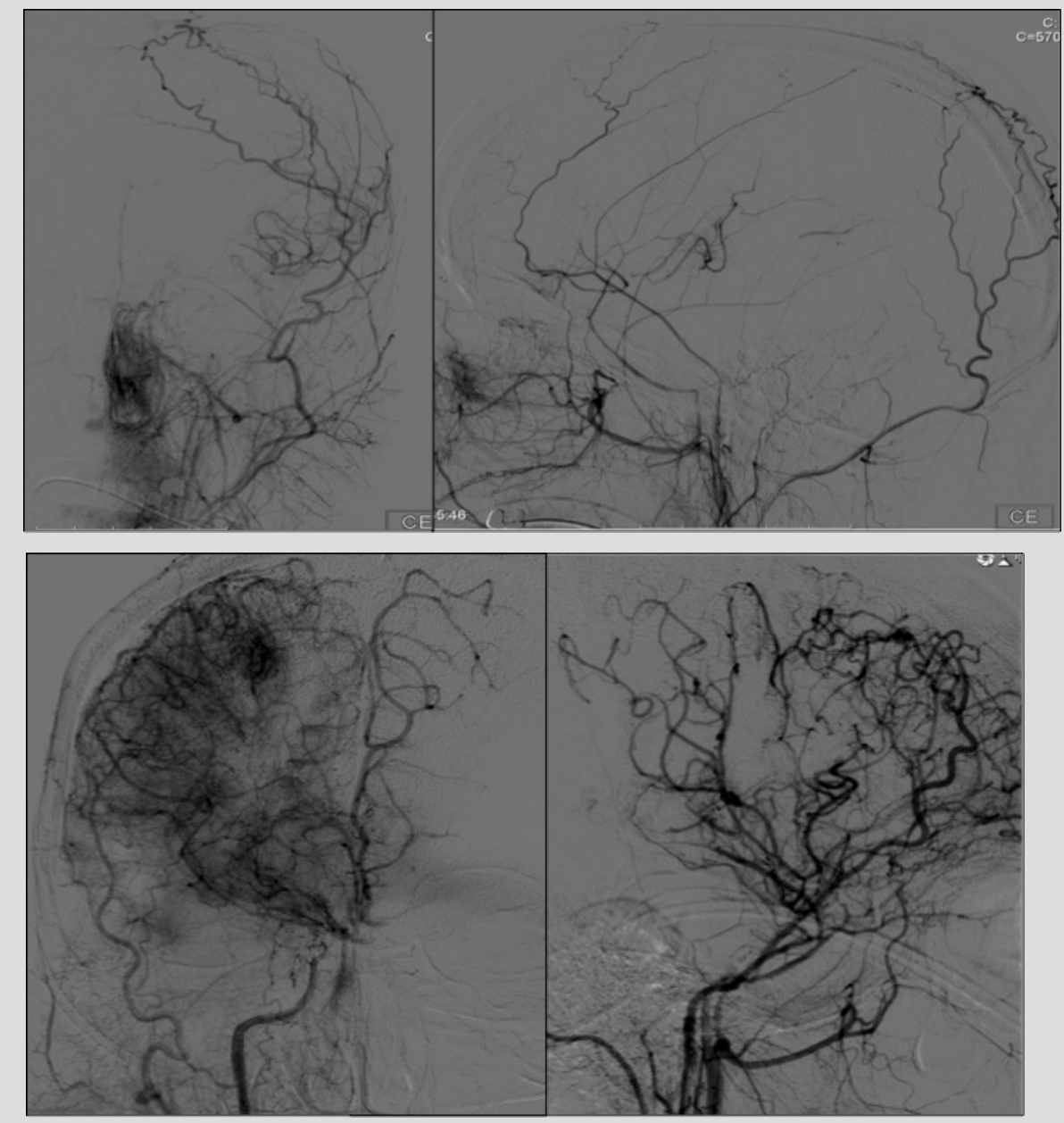

Figure 2. Cerebral angiography showed total occlusion of the left internal carotid artery with multiple leptomeningeal collateral vessels of the left posterior cerebral artery filling retrogradely the territory of the left middle cerebral artery
Figure 3. Cerebral angiography revealed right internal carotid artery occlusion after the emergence of the anterior choroidal artery and an abnormal network of transdural collateral vessels that filled retrogradely the territory of the right middle and anterior cerebral arteries

\section{DISCUSSION}

Moyamoya disease (MMD) is a rare, chronic and progressive vasculopathy characterized by stenosis or occlusion of the intracranial ICA and the proximal branches ${ }^{[1]}$. Despite familial occurrence in approximately $10-15 \%$ of cases, the aetiology of MMD remains unknown ${ }^{[2]}$. The Japanese term "moyamoya" means "puff of smoke" and is a reference to the pathognomonic arteriographic appearance of MMD, corresponding to compensatory development of prominent arterial collateral circulation ${ }^{[3]}$. Traditionally, patients with these characteristic features and no known associated risk factors are considered to have MMD, while those having one of the well-recognized predisposing conditions are classified as having $\mathrm{MMS}^{[4]}$. There are numerous reported links between MMS and a wide variety of diseases, the most frequent and strongly associated conditions being neuro-fibromatosis type 1 , cranial therapeutic radiation, Down syndrome and sickle cell disease $^{[2,4]}$.

Radiation-induced MMS is a serious, well-described and probably frequent, yet under-recognized, late complication of cranial radiotherapy ${ }^{[3]}$. A younger age at radiation exposure seems to increase the risk of developing vasculopathy, with over half of all cases occurring in children under 5 years of age ${ }^{[3,5]}$. Not surprisingly, a parasellar tumour location, such as with craniopharyngiomas, is another risk factor, likely due to the close relationship to the circle of Willis of the tumour ${ }^{[2-5]}$. To prevent it, some authors suggest reduced radiation doses in children with parasellar tumours ${ }^{[5]}$. However, the amount of energy necessary to cause this effect is unknown ${ }^{[1]}$, and the latency between radiation and vasculopathy development may vary from several months to years ${ }^{[4]}$.

Generically, the symptoms may be due to brain ischaemia related to the hypoperfused hemisphere or to the deleterious consequences of the compensatory mechanisms responding to the ischaemia ${ }^{[1]}$. If, on the one hand, the rate of haemorrhage among adults is approximately 7 times higher than the rate among children ${ }^{[1]}$, on the other, MMS accounts for as much as $6 \%$ of childhood strokes ${ }^{[3,4]}$. Thus, MMS should be considered in younger patients presenting with acute neurologic deficits, particularly those treated with cranial radiotherapy. 
Although cerebral angiography is the gold standard for diagnosis of MMS, magnetic resonance imaging (MRI) can be used to detect blood flow disturbances associated with presymptomatic vascular lesions, as well as for a sensitive and quantitative analysis of the vascular changes ${ }^{[4,5]}$. TCD provides a non-invasive method to evaluate intracranial haemodynamics and it has been used in the initial evaluation and to follow up progression of MMS. Disease severity and its angiographic progression is classified into the 6 stages of the Suzuki grading system ${ }^{[1]}$.

Treatment of acute stroke should include intravenous hydration, avoidance of hypotension and administration of supplemental oxygen to avoid hyperventilation and consequent vasoconstriction. Currently, secondary stroke prevention for patients with symptomatic MMS is based on improving blood flow to the affected cerebral hemisphere by surgical revascularization. Two methods for revascularization, direct and indirect, have been described, the latter being the preferred option in younger children ${ }^{[1]}$. Considering medical therapy, the benefit of antiplatelet agents is limited and equivocal and oral anticoagulants are not recommend for long-term treatment ${ }^{[1]}$.

We report a case of MMS arising after radiotherapy 20 years previously for craniopharyngioma, emphasizing the importance of an appropriate understanding of late complications of cranial radio-therapy for long-term patient follow-up. In addition to the strong association between radiotherapy for craniopharyngiomas and MMS, the characteristic angiographic pattern favoured the diagnosis of this condition in our patient. As MMS is usually progressive, early detection and prompt treatment of MMS are both mandatory, as the consequences of delayed treatment are disastrous in young patients, including cognitive and neurologic decline due to repeated strokes.

\section{REFERENCES}

1. Scott RM, Smith ER. Moyamoya disease and moyamoya syndrome. N Engl J Med 2009;360(12):1226-1237.

2. Wu YH, Chang FC, Liang ML, Chen HH, Wong TT, Yen SH, et al. Incidence and long-term outcome of postradiotherapy moyamoya syndrome in pediatric patients with primary brain tumors: a single institute experience in Taiwan. Cancer Med 2016;5(8):2155-2160.

3. Reynolds MR, Haydon DH, Caird J, Leonard JR. Radiation-induced moyamoya syndrome after proton beam therapy in the pediatric patient: a case series. Pediatr Neurosurg 2016:51(6):297-301.

4. Han JY, Choi JW, Wang KC, Phi JH, Lee JY, Chae JH, et al. Coexistence of radiation-induced meningioma and moyamoya syndrome 10 years after irradiation against medulloblastoma: a case report. J Korean Med Sci 2017;32(11):1896-1902.

5. Lee HS, Seol HJ, Kong DS, Shin HJ. Moyamoya syndrome precipitated by cranial irradiation for craniopharyngioma in children. J Korean Neurosurg Soc 2011;50(6):535-537. 